\title{
ON THE MOTION OF TRAPPED PARTICLES
}

\section{IN THE VICINITY OF COROTATION CENTERS}

\author{
C. BEAUGÉ \\ Observatorio Astronomico, Universidad Nacional de Córdoba, \\ Laprida 854, (5000) Córdoba, Argentina \\ and \\ A. LEMAÎTRE and S. JANCART \\ Département de Mathématique, FUNDP, 8 Rempart de la Vierge, \\ 5000 Namur, Belgium
}

\begin{abstract}
In the present paper we analyse the motion of a massless particle during the capture process in an exterior mean-motion resonance under the effects of an external dissipative force. In particular, we study the orbital evolution from its initial approach to the commensurability up to the final nesting place in the periodic orbit around the equilibrium solution.
\end{abstract}

\section{Introduction}

In a recent work (Beaugé et al., 1998; hereafter BLJ98) we presented a new model for the averaged equations of motion of the capture problem, based on a Lie perturbation method (Kamel, 1969) truncated to second order. The application of this model to the case of Stokes drag allowed us to determine the equilibrium solutions of the system (i.e. corotation centers) with a significant increase in precision with respect to previous works.

However, when these results are compared with numerical simulations of the exact equations, we find that the real motion of the particles is not restricted to the equilibrium value but, due to the effects of the short-period terms, will define a periodic orbit around the fixed point. We call such orbit a limit cycle (Poincaré, 1885), and its period is simply the synodic period of the resonance. Fortunately, these cycles may also be reproduced with the second-order model. By means of the inverse transformation to the non-averaged variables we can find approximate analytical expressions for the periodic orbits which, when compared to the numerical simulations, show a very good agreement. Consequently, we currently have a fairly good idea of the final resting place of the trapped particles. The next step is to study the road that takes them there from the initial entrance to the resonance region. In other words, the motion of the body in the vicinity of the limit cycle itself.

This question has received a boost in interest from a recent work by Gomes and Mothe-Diniz (1998). In this paper, the authors performed several studies, via numerical simulations, of the evolution of two different particles (of radii $r$ ) trapped in the same corotation point. They found that, under a wide range of the initial conditions, the relative distance between both bodies diminished exponentially to 
values of the order of machine precision in times of the order of $10^{4}$ years. Their relative velocity also decreased to extremely low values $\left(\sim 10^{-10} \mathrm{~m} / \mathrm{s}\right.$ at $\left.\Delta x \sim 2 r\right)$, which means that these particles experience more a "smooth approach" than an actual collision. These results are of considerable importance to those cosmogonic theories in which resonance trapping is present. Not only do they ensure the actual attraction of different bodies, but they also indicate that any collision will result in accretion. This could help explain how particles of very small size could accrete, even when the mutual gravity is virtually non-existent.

So stated, we wonder whether it would be possible to use our second-order model, together with the expressions for the limit cycles, to obtain approximate analytical solution of the complete non-averaged system during the capture process itself. This is the question we wish to address in this communication.

The manuscript is organized in the following manner: Section 2 presents the problem and reproduces the main results of the second-order theory, together with the expressions for the periodic orbits. Section 3 discusses the linearization of the system around the fixed point and the general solution of the eigensystem. Results and comparisons are presented in Section 4 and conclusions close the paper in Section 5.

\section{The Second-Order Model}

\subsection{STATEMENT OF THE PROBLEM}

As usual, let us suppose the planar elliptic restricted three-body problem in the vicinity of an exterior $(p+q) / p$ mean-motion resonance. The external dissipative force will be modeled by a Stokes drag with $\alpha=0.995$ (Adachi et al., 1976). Let $C$ denote the drag coefficient which has units of unity over time. The set of resonant variables is chosen to be $\left(L, e, \sigma, \sigma_{1}, \sigma_{2}\right)$, where $L=\sqrt{\mu a}$ and the angular variables are defined through

$$
\begin{aligned}
q \sigma & =(p+q) \lambda_{1}-p \lambda-q \varpi \\
q \sigma_{1} & =-(p+q) \lambda_{1} / p+\lambda \\
\sigma_{2} & =\lambda_{1} / p .
\end{aligned}
$$

Here $\mu$ is the gravitational constant, $a$ and $e$ denote the semimajor axis and eccentricity of the body, $\lambda$ stands for the mean longitude and $\varpi$ the longitude of perihelion. Similar quantities, with subindex 1 , hold for the perturbing planet of mass $m_{1}$.

Defining the position vector of this non-canonical set as $x=\left(L, e, \sigma, \sigma_{1}, \sigma_{2}\right)$, the system of differential equations governing the motion of the particle can be 
succinctly written as

$$
\frac{d x}{d t}=\sum_{i_{1}, i_{2}, i_{3}, i_{4}, i_{5}}\left(f_{0}^{(0)}+m_{1} f_{1}^{(0)}\right) L^{i_{1}} e^{i_{2}} E^{\sqrt{-1}\left(i_{3} \sigma+i_{4} \sigma_{1}+i_{5} \sigma_{2}\right)}
$$

where $f_{0}^{(0)}=f_{0}^{(0)}\left(i_{1}\right)$ marks the two-body gravitational contribution and $f_{1}^{(0)}=$ $f_{1}^{(0)}\left(i_{1}, i_{2}, i_{3}, i_{4}, i_{5}\right)$ groups the complete perturbing forces (per unit of $m_{1}$ ), including the dissipative part. Here we have used the notation $E^{\theta}=\exp \theta$. We must note that all the $f_{0}^{(0)}$ and $f_{1}^{(0)}$ coefficients are constant throughout the phase space (see BLJ98 for further details).

\subsection{The AVERAgED SySTEM}

We search for a transformation of variables $W\left(y ; m_{1}, C\right): x \rightarrow y$ to new variables $y=\left(y_{1}, y_{2}, y_{3}, y_{4}, y_{5}\right)=\left(\bar{L}, \bar{e}, \bar{\sigma}, \overline{\sigma_{1}}, \overline{\sigma_{2}}\right)$ such that the new equations of motion do not depend explicitly on the transformed synodic angle $y_{5}$ :

$$
\frac{d y}{d t}=\sum_{i_{1}, i_{2}, i_{3}, i_{4}} \mathcal{A}_{i_{1}, i_{2}, i_{3}, i_{4}} L^{i_{1}} e^{i_{2}} E^{\sqrt{-1}\left(i_{3} \sigma+i_{4} \sigma_{1}\right)} .
$$

Expanding the transformation function $W$ in a power series of the perturbing mass $m_{1}$, we can explicitly write the relationship between both sets of variables as:

$$
x=y+m_{1} W_{1}(y ; C)+\frac{1}{2} m_{1}^{2} W_{2}(y ; C)+\ldots
$$

where the new $W_{i}(y ; C)$ are independent of the planetary mass. Via the Lie transform method (Kamel, 1969; Henrard, 1970) we find that the constant coefficients of (3) can be approximated by

$$
\mathcal{A}_{i_{1}, i_{2}, i_{3}, i_{4}}=f_{0}^{(0)}+m_{1} f_{0}^{(1)}+\frac{1}{2} m_{1} C f_{0}^{(2)}+\ldots
$$

where the new $f_{0}^{(k)}=f_{0}^{(k)}{ }_{i_{1}, i_{2}, i_{3}, i_{4}}$ are obtained through the averaging process itself (Triangle rule). The fixed points of system (3) automatically yield the corotation centers of the averaged system. Denoting these values as $y_{c}=y_{c}\left(m_{1}, C\right)$, we have that $\dot{y}_{c}=0$.

Next, to obtain the limit cycles around $y_{c}$, we must perform the inverse transformation. Retaining only the lowest-order terms, the periodic orbit in the old variables can be written as

$$
x \simeq y_{c}+m_{1} W_{1}\left(y_{c} ; C\right)=y_{c}+\sum_{j} \omega^{(j)} E^{\sqrt{-1}\left(j \sigma_{2}\right)}
$$

where the $\omega^{(j)}=\omega^{(j)}\left(y_{c} ; m_{1}, C\right)$ coefficients are also given by the averaging process. 


\section{Linearization around the Fixed Point}

\subsection{THE EIGENSYSTEM}

The usual way to study the evolution of the system in the vicinity of the periodic orbit is through Floquet theory. In this, we linearize the equations of motion around the periodic orbit itself, determine the monodromy matrix, and search for the eigenvalues of the resulting system at fixed times. However, the steps taken in the averaging process described in the previous section allow us to take a simpler road. Since the averaging process relates the limit cycle in $x$ with the fixed point in $y$, we can directly study the linear system around the corotation center. The solution of the eigensystem (in $y$ ) can then be transformed to the old variables directly by means of equations (6). Thus, we avoid the complications of the linearization around a periodic solution and can work directly on the fixed point (see Hale, 1969). This is the aim of the present section.

Let us define $\xi=y-y_{c}$ and write the linearized system of (3) as $\dot{\xi}=\mathcal{B} \xi$. From (3) the expression for the Jacobian matrix is trivial, since all the coefficients $\mathcal{A}$ are constant and the derivatives can be performed explicitly. Thus, we can write

$$
\mathcal{B}=\sum_{i_{1}, i_{2}, i_{3}, i_{4}} \mathcal{A}_{i_{1}, i_{2}, i_{3}, i_{4}}^{\prime} L_{c}^{i_{1}} e_{c}^{i_{2}} E^{\sqrt{-1}\left(i_{3} \sigma_{c}+i_{4} \sigma_{1 c}\right)}
$$

where $\mathcal{A}^{\prime}$ denote the new coefficients after the differentiation.

Knowing $\mathcal{B}$, we can construct the characteristic equation and solve for the eigenvalues $\lambda_{i}$ and the eigenvectors $\psi_{i}$. Then, the general solution will be given by:

$$
y=y_{c}+\sum_{i=1}^{4} K_{i} \psi_{i} E^{\lambda_{i} t} .
$$

The complex constants $K_{i}$ are determined as solutions of the linear system $\psi K=$ $y_{0}-y_{c}$, where $\psi$ is the matrix of eigenvectors components and $y_{0}$ mark the initial conditions.

\subsection{THE COMPLETE SOLUTION}

It is now possible to introduce the solution (8) of the averaged system into the inverse transformation (6). In order to obtain an explicit expression, we approximate

$$
W_{1}(y) \simeq W_{1}\left(y_{c}\right)+\left(\frac{\partial W_{1}}{\partial y}\right)_{y=y_{c}}\left(y-y_{c}\right) .
$$

Writing the derivative of the transformation function (evaluated at the fixed point) as

$$
\left(\frac{\partial W_{1}}{\partial y}\right)_{y=y_{c}}=\sum_{j} \Omega_{j} E^{\sqrt{-1} j \sigma_{2}}
$$




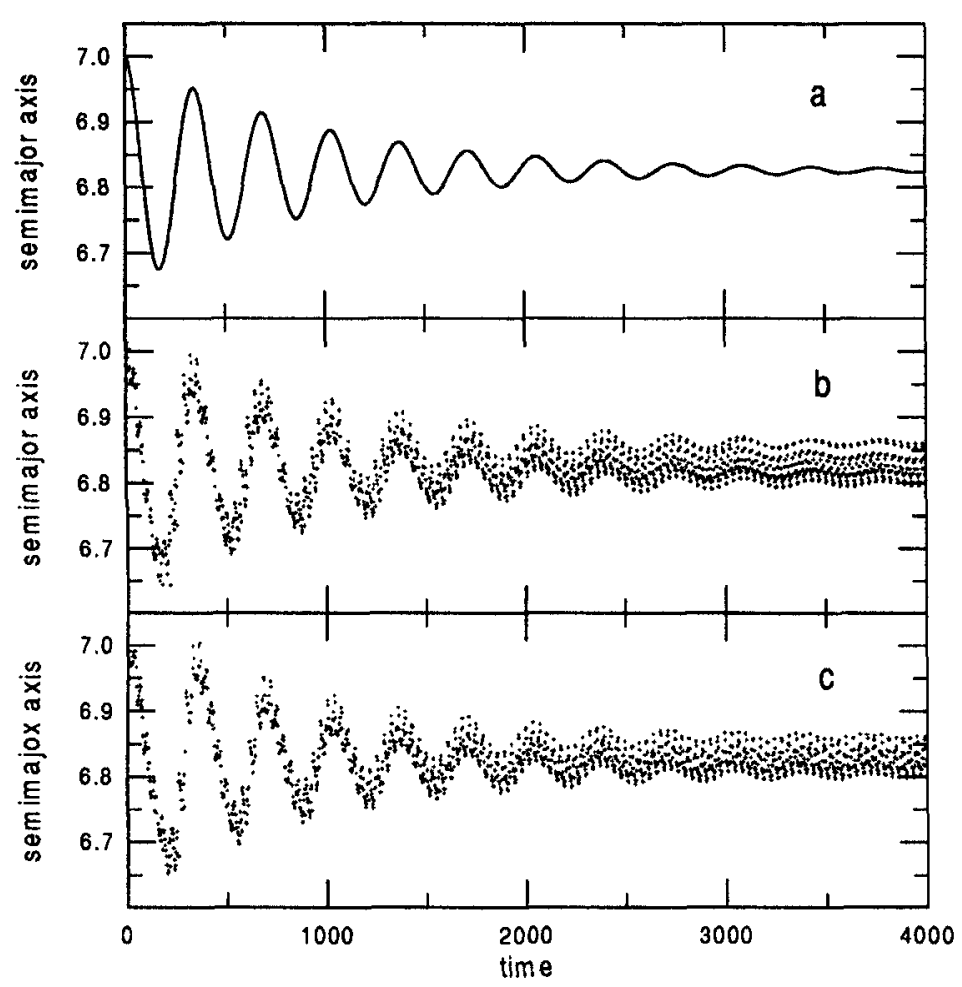

Fig. 1. Temporal evolution of the semimajor axis during the capture of a particle in a corotational solution in the $2 / 3$ resonance. (a) Evolution of the mean semimajor axis (i.e. $y_{1}$ ) according to equation (8). (b) Idem, but for the non-averaged semimajor axis (i.e. $x_{1}$ ) as obtained by equations (11). (c) Numerical simulation of the exact equations.

and remembering expression (8) for $\left(y-y_{c}\right)$, we can finally obtain the solution of the complete system around the limit cycle as

$$
\begin{aligned}
x(t)=y_{c} & +\sum_{i}^{4} K_{i} \phi_{i} E^{\lambda_{i} t}+\sum_{j} \omega^{(j)} E^{\sqrt{-1} j\left(\nu_{2} t+\sigma_{20}\right)} \\
& +\sum_{i, j} \Omega_{j} K_{i} \phi_{i} E^{\left(\lambda_{i} t+j \sqrt{-1}\left(\nu_{2} t+\sigma_{20}\right)\right)}
\end{aligned}
$$

where $\nu_{2}=\sigma_{2}\left(y=y_{c}\right)$ and $\sigma_{20}$ is the initial value of the synodic angle.

\section{Results}

Equation (11) finally gives the evolution of the system variables as a function of time. The value of $y_{c}\left(m_{1}, C\right)$ is determined as zeros of (3) and the initial 

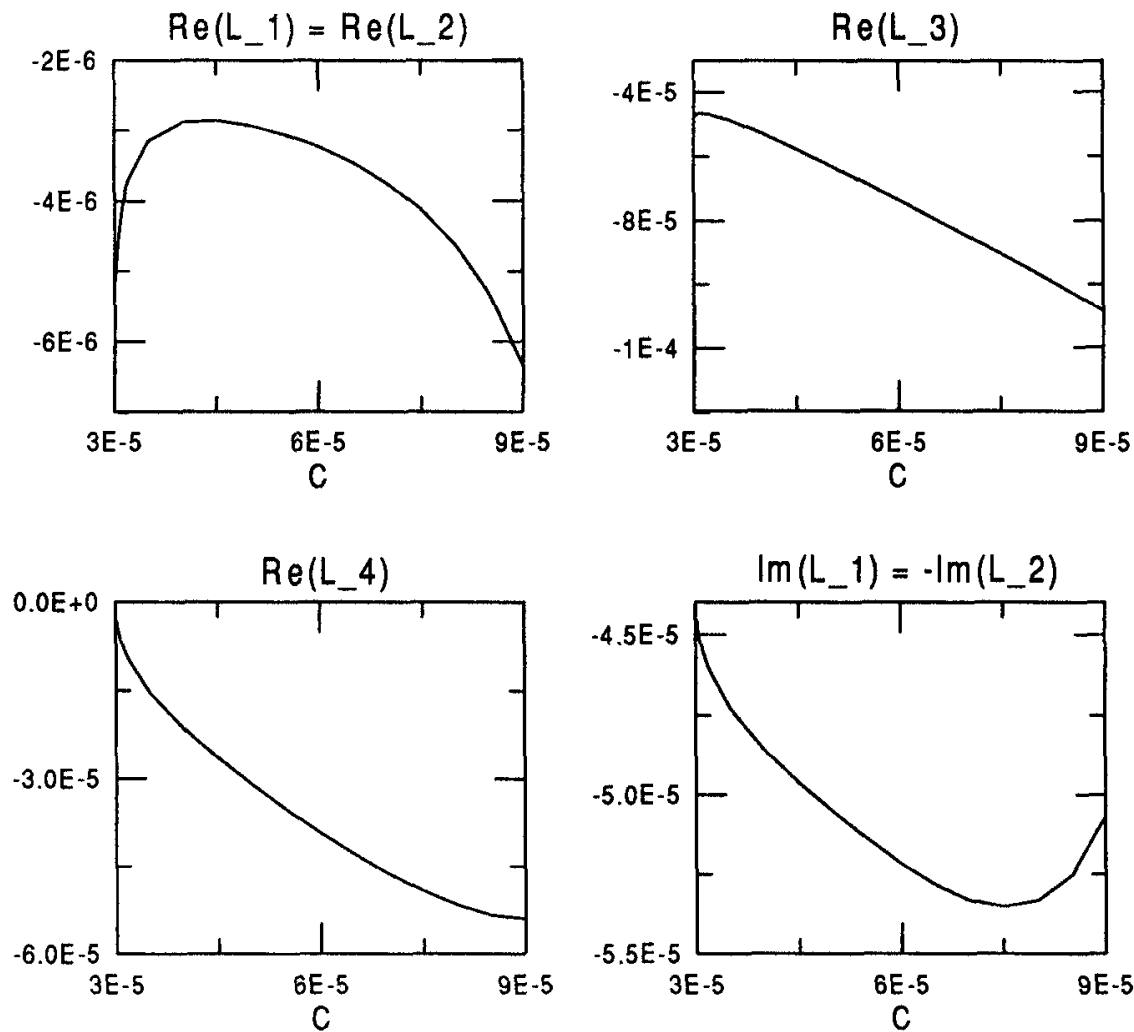

Fig. 2. Real and imaginary parts of the eigenvalues of the variational equations for the $2 / 3$ resonance, as a function of the drag coeffcient $C$.

conditions enter through coefficients $K$. The next step is to compare this model with the numerical results.

We begin with a direct comparison of $x(t)$ with that obtained from numerical simulations. In Figure 1 we present the semimajor axis as a function of time for a body initially located at $a=7$ U.A. On the top graph we show the behaviour of the mean variable, as determined by the averaged solution (8). We notice the damped oscillation and the final corotational value for large values of $t$. Since the eigenvalues $\lambda_{i}$ are such that $\Im\left(\lambda_{1}\right)=-\Im\left(\lambda_{2}\right)$, and $\lambda_{3}$ and $\lambda_{4}$ contain no imaginary parts (i.e. $\Im\left(\lambda_{3}\right)=\Im\left(\lambda_{4}\right)=0$ ), the frequency of oscillation is identical for all the orbital elements. The middle plot in Figure 1 now shows the temporal evolution of the osculating semimajor axis, as determined through (11). This can now be compared with the numerical results, seen in the bottom graph in Figure 1. The agreement between them is very good.

Having tested the analytical solution, we can concentrate our study on the eigen- 

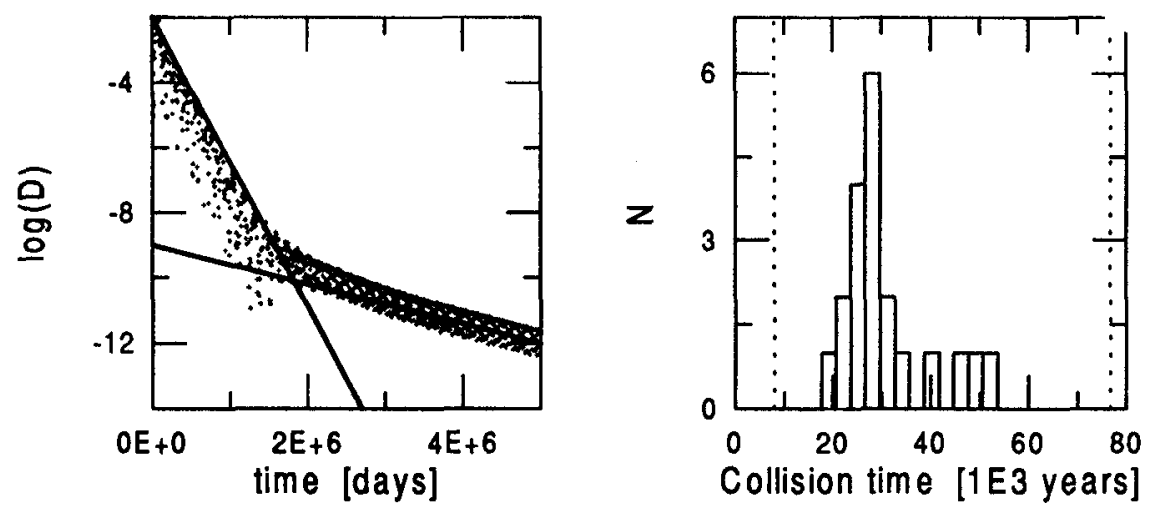

Fig. 3. (a). Logarithm of $\Delta x$ as function of time. Thick lines show linear decrease according to $\lambda_{1}$ (small $t$ ) and $\lambda_{4}$ (large $t$ ). (b). Distribution of collision times $T_{c o l}$ for 20 particles with different initial conditions. $C=3.02 \times 10^{-5}$ in both cases.

values and their relationship with the convergence rate of nearby orbits. Figure 2 shows the variation of $\lambda_{i}$ as a function of the drag coefficient $C$ for the $2 / 3$ resonance. As was mentioned in the previous paragraph, the first two values are complex conjugates and contain the only non-zero imaginary parts. Thus, any solution of the linearized system will display a damped oscillation with a single fundamental frequency in all coordinates. This frequency will be given by $\Im\left(\lambda_{1}\right)$ which, in principle, has no relationship at all with the inverse of the synodic period. Hence, the final limit cycle will only become evident for $t>>-1 / \Re\left(\lambda_{1}\right)$. Second, we can see that $\lambda_{4} \rightarrow 0^{-}$as $C \rightarrow C^{*} \simeq 3.2 \times 10^{-5}$. This critical value of the drag coefficient marks the end of the corotational regime and the beginning of the libration zone. All capture with $C<C^{*}$ will thus evolve asymtotically to periodic motions in the averaged system, and not to point attractors.

Next, we can focus on the orbital convergence itself, as described in Gomes and Mothe-Diniz (1998). Although exponential convergence of nearby orbits is expected from the stability condition of the equilibrium solutions, the present model can give us additional information, such as the expected accretional timescale $T_{c o l}$ for a given population of planetesimals and its dependence on the drag coefficient or with the initial conditions. Let us then suppose two particles of equal radius $r$ (and thus equal drag coefficient $C$ ) and on initial conditions $x(t=0)$ and $x^{\prime}(t=0)$ chosen such that both are captured in the same corotational solution. Then, from equations (8)-(11), and once we have eliminated all periodic modulations, we can estimate the vectorial difference between them as

$$
x(t)-x^{\prime}(t) \equiv \Delta x(t) \propto \sum_{i}^{4} \Lambda_{i} E^{\lambda_{i} t}
$$

where the coefficients $\Lambda_{i}$ are function of the initial conditions of both particles. 


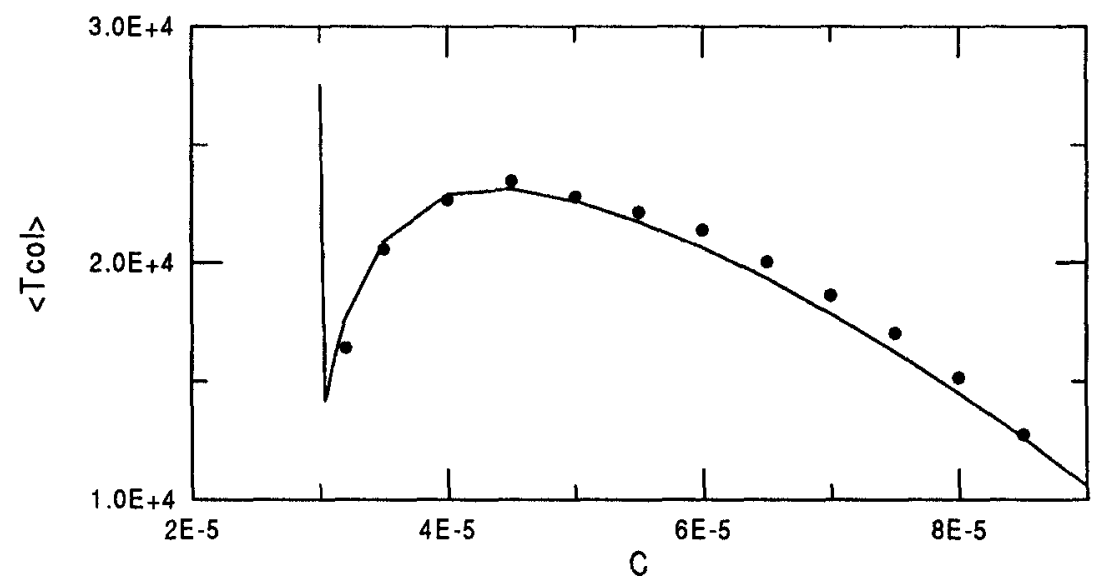

Fig. 4. Real and imaginary parts of the eigenvalues of the variational equations for the $2 / 3$ resonance, as a function of the drag coefficient $C$.

From this expression we can see that the convergence is proportional to four exponentials, each weighted by coefficients that depend on the initial conditions. Now, even though for small timescales the different terms may be of similar magnitude, for extremely large values of $t$ the temporal evolution of $\Delta x(t)$ will tend asymtotically to an exponential proportional to the smallest eigenvalue. In other words, $\Delta x(t) \propto E^{\min \left(\lambda_{i}\right) t}$ (see Fig. 3a).

Thus it seems that, independently of the initial conditions, the convergence rate is given by the lowest eigenvalue of the system. However, this is valid for $t \rightarrow \infty$ (which implies $\Delta x \rightarrow 0$ ) but not necessarily true for finite values of $t$ corresponding to a collision between both bodies (i.e. $\Delta x=2 r$ ). If $T_{\text {col }}$ is sufficiently small, the convergence rate will no longer be unique but function of the initial separations. In order to see this in more detail, we once again make use of equation (12). With it, we can simulate a variation of initial conditions via modifications of the numerical values of $\Lambda_{i}$, and determine collision times in each case.

This calculation was performed for 20 different sets of coefficients and the resulting distribution of $T_{c o l}$ is shown in Figure $3 \mathrm{~b}$. In broken vertical lines we also show the theoretical times if only $\lambda_{4}$ (left line) and $\lambda_{1}$ (right line) where present. $\lambda_{3}$ is too large and can be ignored in the model. We can see that the real values lie between both limits and a certain tendency is noted towards faster accretion. Nevertheless, the dispersion is very significant, covering more than half an order of magnitude. From a cosmogonical point of view this result may be of importance, since it means that even though a swarm of equal-massed particles will in fact converge towards the same corotation point, there is no unique timescale for the accretion process.

Notwithstanding this last fact, it is possible to determine a mean collision time 
$\left\langle T_{\text {col }}\right\rangle$, defined as the average over the different $\lambda_{i}$ (see (12)). Even though individual times may vary, this quantity can be thought as a characteristic timescale of the system for the given drag coefficient. The result of this calculation, as a function of $C$, is shown as a continuous line in Figure 4. Notice the steep increase in collision times for $C$ close to the librational limit ( $T_{\text {col }}$ at this point). For comparison with numerical results of the exact equations, for each value of $C$ we took 20 different initial conditions and determined directly $\left\langle T_{\text {col }}\right\rangle$. These were then plotted in full circles. We can see that the agreement with the analytical results is very good.

\section{Acknowledgements}

This work is part of collaboration initiated during C. Beaugé's one month visit to Namur. The authors are grateful to the FUNDP for the partial financing of the stay. Additional support from CONICET, CONICOR and Secyt/UNC is also greatly appreciated.

\section{References}

Adachi, I., Hayashi, C., and Nakazawa, K.: 1976, Prog.Theor.Phys., 56, 1756-1771.

Beaugé, C., Lemaître, A. and Jancart, S.: 1998, Planet. Space Science, in press.

Gomes, R. and Mothe-Diniz, T.: 1998, in preparation.

Hale, J.K.: 1969), Ordinary Differential Equations, Wiley-Interscience, J. Wiley \& Sons, New York. Henrard, J.: 1970, Celest. Mech., 3, 107-120.

Kamel, A.A:. 1969, Celest. Mech., 1, 190-199.

Poincaré, H.: 1885, J. de Math. pures appl., ser. 4, 1, 167. Reprinted in Oeuvres de Henri Poincaré, I, 90, Gauthier-Villars, Paris, (1928). 